\title{
The novo Corona virus 2019
}

\section{Introduction}

Corona viruses are a large family of viruses that cause diseases ranging from the common cold to more serious conditions, such as the Middle East Respiratory Syndrome (MERS-CoV), Severe Acute Respiratory Syndrome (SARS-CoV) and the New Corona Virus of 2019 (COVID-19).

The pandemic (epidemic disease that spreads to many countries or that attacks almost all the individuals of a locality or region) that currently affects the planet Earth begins in the province of Wuhan, China, on December 31, 2019.

\section{Transmission}

The animal that serves as a reservoir from which the microorganism spreads to humans is unknown. It is believed that the virus is transmitted mainly from person to person, especially among those who are in close contact with each other (in a diameter of 6 feet), through respiratory drops produced when a patient coughs or sneezes. These droplets can fall into the mouth or nose of those who are nearby and be inhaled into the lungs.

A person can get COVID-19 by touching a surface or object contaminated with the germ and then touching their mouth, nose or eyes.

The virus is believed to be more contagious when the patient is more symptomatic (sickest). There is a possibility that it will spread even when the individual has no symptoms (pre-symptomatic).

\section{Prevention}

There is currently no vaccine to avoid COVID-19. The best way to prevent the disease is not to be exposed to it. Nowadays the recommendations that are made to avoid the contagion with this virus are: to take the general measures for all respiratory illness, refrain from patients who suffer it, to avoid traveling to the countries where there is currently a high virus alert; These are Mainland China, South Korea, Japan, Singapore, Italy, Spain and Australia.

Be careful not to have close contact with people who have symptoms and have visited these countries. Do not touch your eyes, nose or mouth, cover your cough and sneeze with disposable paper and throw it in the trash after each use, stay home if you have symptoms and call your doctor. Clean and disinfect frequently used objects and surfaces with alcohol-based sterilizing products. If you have symptoms or reside in a city where there have been cases, wear a mask. Wash your hands with soap for a minimum of 20 seconds, especially after using the bathroom, before eating, when you shake hands, blow your nose, cough or sneeze; If you do not have soap and water available, you can use disinfection products that contain a minimum of $60 \%$ alcohol.

\section{Symptom}

The symptoms of COVID-19 are: cough, fever and shortness of breath. Cases have also been reported in which there are a sore throat and diarrhea, which will appear two to fourteen days after being exposed to the disease. These mild initial symptoms may progress to pneumonia, leading to respiratory failure and death. The elderly and people with chronic medical conditions may have an increased risk of
Volume 8 Issue 2 - 2020

\section{Victor Garrido MD}

Sharing Hope Foundation, USA

Correspondence: Victor Garrido, Sharing Hope Foundation, 9405 Springdale Dr. Raleigh, North Carolina, USA, Email dr.victorgarrido@gmail.com

Received: March 16,2020| Published: April 15, 2020

suffering from the most serious variant of the disease.

\section{Treatment}

There is no specific antiviral for COVID-19. Those who suffer from it should be treated to improve the symptoms they present (symptomatic treatment). In severe cases, symptomatic management should be complemented with supportive therapy to vital organs.

It is only a matter of time before COVID-19 arrives in the Dominican Republic due to multiple conditions, including tourism. Today we ask:

Do we have devices in ports and airports to prevent the entry of infected people? Are instruments being used to detect the body temperature of several people simultaneously?

Is there a team at ports and airports that ask travelers if they have specific products that can transport the COVID-19?

Is a questionnaire delivered on airplanes where the passenger answers YES / NO to questions such as whether they have visited or made a stop in any of the countries mentioned above, or if they have presented symptoms?

Are there areas of isolation in all the entrance doors to our country?

Has health personnel been designated and trained by the Ministry of Public Health and Social Assistance? Do we have the biosecure equipment to handle the cases of COVID-19 at the entrance doors?

Was a preventive public education campaign launched on radio, television, social networks, etc.?

Were reference hospitals designated and management guidelines established for COVID-19?

Finally, we emphasize the importance of public and individual prevention because of its cost / effectiveness. We call the attention of the authorities to start urgent preventive actions given the seriousness of the threat of COVID-19. We cannot afford to continue exercising a reactive and non-preventive health policy.

\section{Acknowledgments}

Mrs. Rocío Garrido for her edition.

\section{Conflicts of interest}

Author declares that there is no conflict of interest. 